\title{
Collapse of charge ordering and enhancement of magnetocaloric effect in nanocrystalline $\mathrm{La}_{0.35} \mathrm{Pr}_{0.275} \mathrm{Ca}_{0.375} \mathrm{MnO}_{3}$
}

\author{
M. H. Phan, ${ }^{1, a)}$ S. Chandra, ${ }^{1}$ N. S. Bingham, ${ }^{1}$ H. Srikanth, ${ }^{1, b)}$ C. L. Zhang, ${ }^{2}$ S. W. Cheong, ${ }^{2}$ \\ T. D. Hoang, ${ }^{3}$ and H. D. Chinh ${ }^{3}$ \\ ${ }^{1}$ Department of Physics, University of South Florida, Tampa, Florida 33620, USA \\ ${ }^{2}$ Department of Physics and Astronomy and Rutgers Center for Emergent Materials, Rutgers University, \\ Piscataway, New Jersey 08854, USA \\ ${ }^{3}$ Department of Inorganic Chemistry, Hanoi University of Technology, 1 Dai Co Viet Str., Hanoi, Vietnam
}

(Received 23 July 2010; accepted 22 November 2010; published online 13 December 2010)

\begin{abstract}
We demonstrate the possibility of enhancing both the magnetocaloric effect (MCE) and refrigerant capacity (RC) in nanostructured mixed phase manganites. A comparative study of the magnetic and magnetocaloric properties of $\mathrm{La}_{0.35} \mathrm{Pr}_{0.275} \mathrm{Ca}_{0.375} \mathrm{MnO}_{3}$ in single crystalline and nanocrystalline forms is presented. While the conventional trend is reduction of magnetization and MCE with nanostructuring, we show that the opposite is true in the case of mixed phase manganites. The charge-ordered state is largely suppressed and ferromagnetic order is established in the nanocrystalline sample with an average particle size of $50 \mathrm{~nm}$. Consequently, a strong enhancement of MCE and RC and a strong reduction of thermal and field hysteresis losses are achieved in the nanocrystalline sample. This finding opens up a way of exploring magnetic refrigerant materials at the nanometer scale for active magnetic refrigerators. (C) 2010 American Institute of Physics.
\end{abstract}

[doi:10.1063/1.3526380]

Magnetic refrigeration is a cooling technology based on the magnetocaloric effect (MCE). ${ }^{1}$ It has several advantages (e.g., higher efficiency, compactness, and environmental safety) over conventional gas compression techniques. Current research is focused on seeking materials that are costeffective and exhibit large refrigerant capacity $(\mathrm{RC})$, which is a measure of the amount of heat transfer between the cold and hot sinks in an ideal refrigeration cycle. ${ }^{2-4}$ The RC depends not only on the magnitude of magnetic entropy change $\left(\Delta \mathrm{S}_{\mathrm{M}}\right)$, but also on its temperature dependence [e.g., the full width at half maximum of the $\Delta S_{M}(T)$ peak.$^{5,6}$ In this context, magnetic nanoparticles are more desirable than their bulk counterparts, because the particle size distribution and interparticle interactions have been shown to broaden $\Delta \mathrm{S}_{\mathrm{M}}(\mathrm{T})$ over a wide temperature range thus enhancing $\mathrm{RC}^{7-9}$ In addition, magnetic nanoparticles can easily be assembled as thin films through self-assembly, which are essential for applications of a cooling device for microelectromechanical systems (MEMS) and nanoelectromechanical systems (NEMS). ${ }^{8,9}$ However, a significant reduction in $\Delta \mathrm{S}_{\mathrm{M}}$ is often obtained in the nanoparticle sample, ${ }^{7,9}$ resulting mainly from the significant reduction of the magnetization on size reduction, due to the loss of long-range ferromagnetic order and the large local magnetic disorder on surface of the nanoparticles. This tends to negate the promise of enhanced RC due to the broad MCE over a large temperature range. Thus it continues to remain challenging to enhance both the $\Delta \mathrm{S}_{\mathrm{M}}$ and $\mathrm{RC}$ in magnetic nanoparticle systems in spite of their desired properties.

In this letter, we demonstrate the strong enhancement of $\Delta \mathrm{S}_{\mathrm{M}}$ and $\mathrm{RC}$ and the strong reduction of hysteretic losses in charge-ordered $\mathrm{La}_{0.35} \mathrm{Pr}_{0.275} \mathrm{Ca}_{0.375} \mathrm{MnO}_{3}$ upon size reduction to the nanometer scale $(50 \mathrm{~nm})$. This finding provides an

\footnotetext{
a) Electronic mail: phanm@usf.edu.

${ }^{b)}$ Electronic mail: sharihar@usf.edu.
}

opportunity for developing nanoscale magnetic refrigerant materials for active magnetic refrigerators.

$\mathrm{La}_{5 / 8-\mathrm{y}} \mathrm{Pr}_{\mathrm{y}} \mathrm{Ca}_{3 / 8} \mathrm{MnO}_{3}$ with $\mathrm{y}=0.275$ was chosen for the purpose of this study, since it possesses coexisting low temperature ferromagnetic (FM) and high-temperature charge ordering $(\mathrm{CO})$ states that could vary on size reduction. ${ }^{10}$ The single crystalline samples (referred to as bulk henceforth in the paper) were synthesized in an optical floating-zone furnace. The nanocrystalline samples (referred to as nano) were prepared by sol-gel method. Lanthanum, praseodymium, calcium, and manganese nitrate as raw materials were of analytical grade and were used as starting materials without further purification. These solutions were mixed and citric acid was used as a complex activity with these metal cations. Gel was get at $100{ }^{\circ} \mathrm{C}$ and drying at $120{ }^{\circ} \mathrm{C}$. This gel was heated at $500{ }^{\circ} \mathrm{C}$ for $3 \mathrm{~h}$ in oxygen flow in order to decompose and burn the almost organic compounds. Finally, the nanocrystalline samples were obtained after annealing at $850{ }^{\circ} \mathrm{C}$ for $7 \mathrm{~h}$ in a flow of oxygen gas. The x-ray diffraction (XRD) confirmed the phase purity of the samples. The particle size of

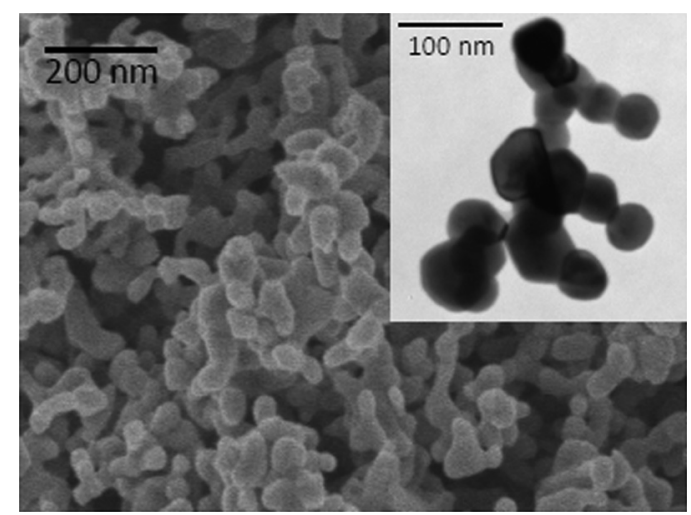

FIG. 1. A SEM image of nanocrystalline $\mathrm{La}_{0.35} \mathrm{Pr}_{0.275} \mathrm{Ca}_{0.375} \mathrm{MnO}_{3}$. The inset is a TEM image of this sample. 


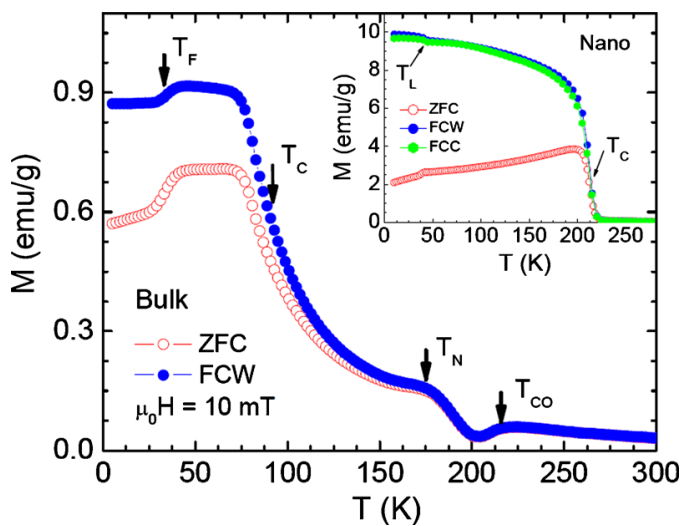

FIG. 2. (Color online) ZFC and FC magnetization data taken at a $10 \mathrm{mT}$ applied field for the bulk and nanocrystalline $\mathrm{La}_{0.35} \mathrm{Pr}_{0.275} \mathrm{Ca}_{0.375} \mathrm{MnO}_{3}$ samples.

the nanocrystalline sample was determined to be about $50 \pm 5 \mathrm{~nm}$ by XRD, scanning electron microscopy (SEM), and transmission electron microscopy (TEM). A typical SEM image along with TEM for the nanocrystalline sample is shown in Fig. 1. Magnetic and magnetocaloric measurements were conducted using a commercial physical property measurement system from Quantum Design with a temperature range of 5-300 K and applied fields up to $7 \mathrm{~T}$.

Figure 2 and its inset display the temperature dependence of zero-field-cooled (ZFC) and field-cooled (FC) magnetization taken at a field of $10 \mathrm{mT}$ for the bulk sample and for the nanocrystalline sample, respectively. Clearly, the bulk
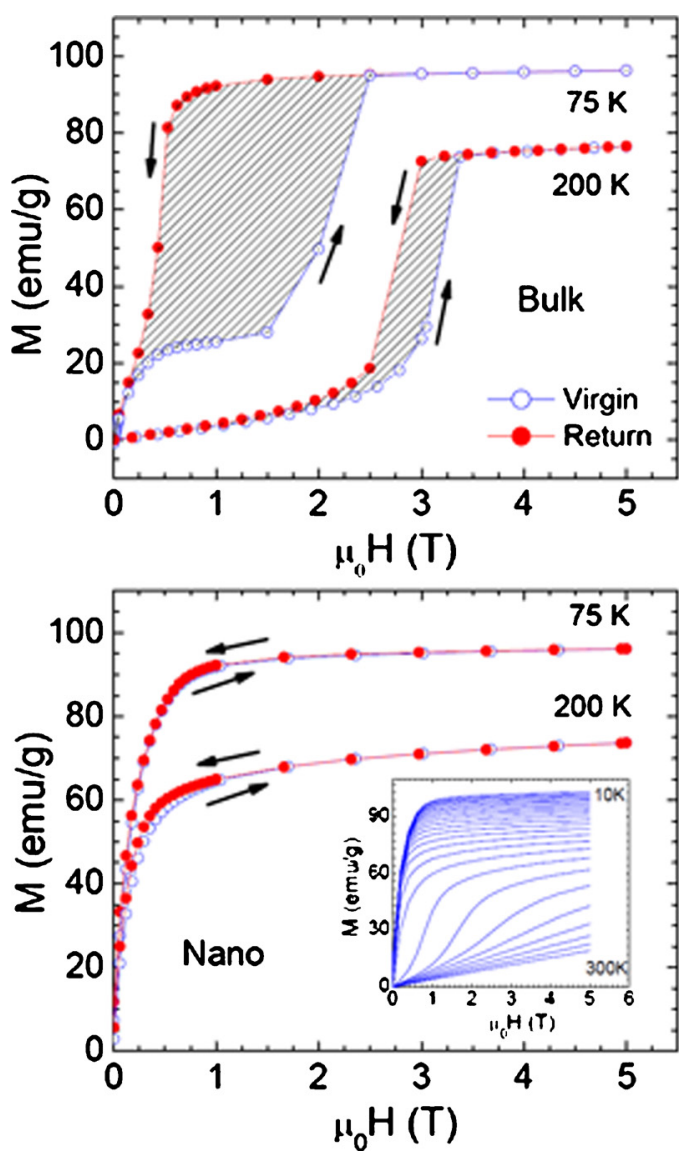

FIG. 3. (Color online) The hysteresis loops taken at 200 and $75 \mathrm{~K}$ for the bulk (a) and nano (b) samples. The inset shows a family of isothermal magnetization curves taken at different fixed temperatures between 10 and $300 \mathrm{~K}$ for the nanocrystalline sample.

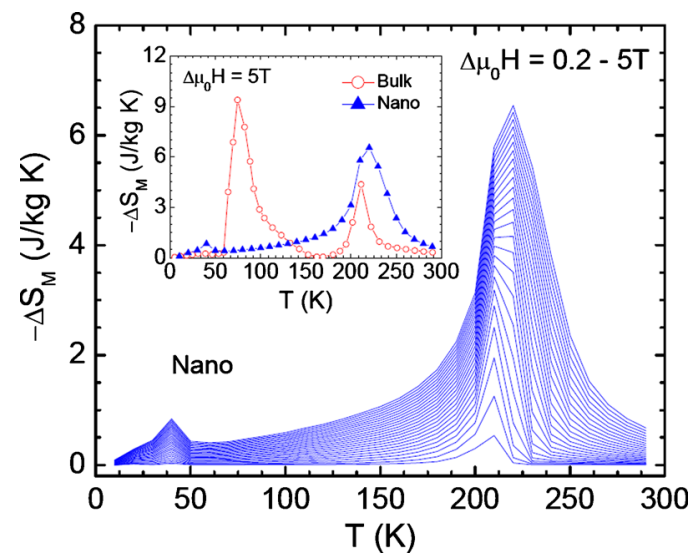

FIG. 4. (Color online) Temperature dependence of magnetic entropy change $-\Delta \mathrm{S}_{\mathrm{M}}$ at different applied fields up to $5 \mathrm{~T}$ for the nanocrystalline sample. The inset shows the temperature dependence of $-\Delta \mathrm{S}_{\mathrm{M}}$ for $\Delta \mu_{0} \mathrm{H}=5 \mathrm{~T}$ for the bulk and nano samples.

sample undergoes multiple magnetic transitions. A peak at $\mathrm{T}_{\mathrm{CO}} \sim 210 \mathrm{~K}$ is due to the $\mathrm{CO}$ transition, and a shoulder at a lower temperature, $\mathrm{T}_{\mathrm{N}} \sim 175 \mathrm{~K}$, arises from the antiferromagnetic (AFM) order. As $\mathrm{T}$ is further decreased, the magnetization sharply increases and an FM transition is observed at $\mathrm{T}_{\mathrm{C}} \sim 75 \mathrm{~K} .{ }^{10,11}$ However, the case is very different for the nano sample, which undergoes a paramagnetic to ferromagnetic (PM-FM) transition at $\mathrm{T}_{\mathrm{C}} \sim 215 \mathrm{~K}$ followed by a small jump in the magnetization at a low temperature of $\sim 45 \mathrm{~K}$ (see inset of Fig. 2). This implies that the CO state is largely suppressed and the FM order is established in the nano sample. ${ }^{12-14}$ To check for the presence of any thermal hysteresis in the PM-FM transition region for the nano sample, we measured the FC magnetization both while heating and cooling the specimen. As one can see clearly in inset of Fig. 2 , the nano sample possesses relatively small thermal hysteresis (below $1 \mathrm{~K}$ ), which is desirable for active magnetic refrigeration (AMR).

Figures 3(a) and 3(b) show the M-H loops that illustrate the field hysteresis losses of the bulk and nano samples. The hysteretic losses are determined by computing the hatched area inside each M-H curve. It is observed in Fig. 3(a) that just below the $\mathrm{T}_{\mathrm{CO}}$ the bulk sample exhibits relatively large field hysteresis losses, which become more pronounced with lowering temperature. This may be reconciled with the fact that below the $\mathrm{T}_{\mathrm{CO}}$ both CO/AFM and FM phases coexist and that the FM phase develops with lowering temperature. ${ }^{10}$ The largest hysteretic losses observed around the first-order FM transition $\left(\mathrm{T}_{\mathrm{C}} \sim 75 \mathrm{~K}\right)$ arise mainly from the strongest phase separation that occurs at this temperature. ${ }^{10,11}$ The field hysteresis losses of the bulk sample are greatly reduced in the nano sample [Fig. 3(b)], which is desirable for AMR. ${ }^{5,6}$ Another remarkable feature in Fig. 3 is that at $200 \mathrm{~K}$ (which is just below the $\mathrm{T}_{C O}$ for the bulk sample and just below the $\mathrm{T}_{\mathrm{C}}$ for the nano sample), the low-field magnetization (below $2.5 \mathrm{~T}$ ) is much larger for the nano sample than for the bulk sample. This clearly indicates the enhancement of the magnetization in the nano sample.

Further evidence is seen in the inset of Fig. 3(b) from the isothermal M-H curves for the nano sample. Clearly, the large variations in the magnetization are achieved around the $\mathrm{T}_{\mathrm{C}}$ at low magnetic fields $(<2 \mathrm{~T})$. This is desirable for domestic applications of magnetic refrigerators, where, instead of using "expensive" superconducting magnets, "inexpen- 
TABLE I. Maximum entropy change, $\left|\Delta \mathrm{S}_{\mathrm{M}}^{\max }\right|$, and refrigerant capacity at the peak temperature, $\mathrm{T}_{\mathrm{p}}$, for the bulk and nanocrystalline $\mathrm{La}_{0.35} \mathrm{Pr}_{0.275} \mathrm{Ca}_{0.375} \mathrm{MnO}_{3}$ and other magnetocaloric materials, within the similar temperature range.

\begin{tabular}{|c|c|c|c|c|c|}
\hline Material & $\begin{array}{l}\mathrm{T}_{\mathrm{p}} \\
(\mathrm{K})\end{array}$ & $\begin{array}{c}\Delta \mu_{0} \mathrm{H} \\
(\mathrm{T})\end{array}$ & $\begin{array}{l}\left|\Delta \mathrm{S}_{\mathrm{M}}^{\max }\right| \\
(\mathrm{J} / \mathrm{kg} \mathrm{K})\end{array}$ & $\begin{array}{c}\mathrm{RC} \\
(\mathrm{J} / \mathrm{kg})\end{array}$ & Reference \\
\hline $\mathrm{La}_{0.35} \mathrm{Pr}_{0.275} \mathrm{Ca}_{0.375} \mathrm{MnO}_{3}$ (bulk) & 210 & 5 & 4.5 & 34.64 & Present \\
\hline $\mathrm{La}_{0.35} \mathrm{Pr}_{0.275} \mathrm{Ca}_{0.375} \mathrm{MnO}_{3}(50 \mathrm{~nm})$ & 215 & 5 & 6.2 & 225.6 & Present \\
\hline $\mathrm{La}_{0.215} \mathrm{Pr}_{0.41} \mathrm{Ca}_{0.375} \mathrm{MnO}_{3}$ (bulk) & 210 & 5 & 5.3 & $143.1^{\mathrm{a}}$ & 11 \\
\hline $\mathrm{Na}_{0.5} \mathrm{Ca}_{0.5} \mathrm{MnO}_{3}$ (bulk) & 225 & 6 & 3.45 & $86.3^{3}$ & 15 \\
\hline $\operatorname{Pr}_{0.65}\left(\mathrm{Ca}_{0.7} \mathrm{Sr}_{0.3}\right)_{0.35} \mathrm{MnO}_{3}$ (bulk) & 215 & 7 & 7.8 & $273^{\mathrm{a}}$ & 12 \\
\hline $\mathrm{Pr}_{0.65}\left(\mathrm{Ca}_{0.7} \mathrm{Sr}_{0.3}\right)_{0.35} \mathrm{MnO}_{3}(67 \mathrm{~nm})$ & 225 & 5 & 6.0 & $180^{\mathrm{a}}$ & 13 \\
\hline $\mathrm{La}_{0.67} \mathrm{Ca}_{0.33} \mathrm{MnO}_{3}$ (bulk) & 250 & 1 & 5.1 & $56.2^{\mathrm{a}}$ & 7 \\
\hline $\mathrm{La}_{0.67} \mathrm{Ca}_{0.33} \mathrm{MnO}_{3}(60 \mathrm{~nm})$ & 250 & 1 & 1.75 & $33.5^{\mathrm{a}}$ & 7 \\
\hline $\mathrm{La}_{0.35} \mathrm{Pr}_{0.275} \mathrm{Ca}_{0.375} \mathrm{MnO}_{3}(50 \mathrm{~nm})$ & 215 & 1 & 2.94 & 37.2 & Present \\
\hline
\end{tabular}

${ }^{\mathrm{a}}$ The RC calculated without subtracting hysteretic losses. The RC values of these bulk samples will be significantly reduced after subtracting hysteretic losses, as they undergo a first-order magnetic transition.

sive" permanent magnets can be used as a magnetic field source. ${ }^{1}$ Remarkably, around the $\mathrm{T}_{\mathrm{C}}$ the nano sample shows $S$-shape magnetization, which is typical for metamagnetic materials. ${ }^{6}$ This feature, coupled with the M-T (Fig. 2) and $\mathrm{M}-\mathrm{H}$ (Fig. 3) data, clearly suggests that the $\mathrm{CO}$ volume fraction is much smaller in the nano sample relative to its bulk counterpart, and as a result, a low magnetic field is sufficient to melt the $\mathrm{CO}$ and convert it into the FM state. ${ }^{12-14}$

Finally, the $\Delta \mathrm{S}_{\mathrm{M}}$ of the bulk and nano samples was calculated from a family of the isotherms [see inset of Fig. $3(\mathrm{~b})$, for example] using the Maxwell relation, $\Delta \mathrm{S}_{\mathrm{M}}$ $=\mu_{0} \int(\partial \mathrm{M} / \partial \mathrm{T})_{\mathrm{H}} \mathrm{dH}$, where $\mathrm{M}$ is the magnetization, $\mathrm{H}$ is the magnetic field, and $\mathrm{T}$ is the temperature. ${ }^{1}$ The temperature dependence of $-\Delta S_{M}$ at different magnetic field changes up to $5 \mathrm{~T}$ for the nano sample is plotted in Fig. 4. For comparison, the temperature dependence of $-\Delta \mathrm{S}_{\mathrm{M}}$ for $\Delta \mu_{0} \mathrm{H}=5 \mathrm{~T}$ for the bulk and nano samples is shown in inset of Fig. 4. Interestingly, the large $-\Delta S_{M}$ is sustained over a wide temperature range around the $\mathrm{T}_{\mathrm{C}}$ for the nano sample. In comparison with the bulk sample within the same temperature range (around $210 \mathrm{~K}$ ), the magnitude of $-\Delta \mathrm{S}_{\mathrm{M}}$ is larger and the $-\Delta S_{M}(T)$ curve is much broader for the nano sample (see inset of Fig. 4). Therefore, a much larger RC is expected for the nano sample. To confirm this, the RC of both the bulk and nano samples was calculated as $\mathrm{RC}=\int \Delta \mathrm{S}_{\mathrm{M}}(\mathrm{T}) \mathrm{dT}{ }^{6}$. For $\Delta \mu_{0} \mathrm{H}=5 \mathrm{~T}$, the RC calculated for the nano sample for the case around the $\mathrm{T}_{\mathrm{C}}(\sim 215 \mathrm{~K})$ is $225.06 \mathrm{~J} / \mathrm{kg}$, while for the bulk sample they are $61.28 \mathrm{~J} / \mathrm{kg}$ for the case around the $\mathrm{T}_{\mathrm{CO}}$ $(\sim 210 \mathrm{~K})$ and $187.96 \mathrm{~J} / \mathrm{kg}$ for the case around the $\mathrm{T}_{\mathrm{C}}$ $(\sim 75 \mathrm{~K})$. As noted in previous studies, ${ }^{5,6}$ because the field hysteresis losses are the costs in energy to make one cycle of the magnetic field, they must be considered when calculating the $\mathrm{RC}$ of a magnetic refrigerant material being subjected to field cycling. Therefore, we have subtracted the average hysteretic losses from the RC values calculated without accounting for the hysteretic losses (Fig. 3). The RC after subtracting the average hysteretic losses for the nano sample $(225.06 \mathrm{~J} / \mathrm{kg})$ is about seven times and three times larger than for the bulk sample for the cases around the $\mathrm{T}_{\mathrm{CO}}(34.64 \mathrm{~J} / \mathrm{kg})$ around the $T_{C}(77.43 \mathrm{~J} / \mathrm{kg})$, respectively. The noted drop of the RC after subtracting the average hysteretic losses for the bulk sample for the case around the $\mathrm{T}_{\mathrm{C}}$ (decreases from 187.96 to $77.43 \mathrm{~J} / \mathrm{kg}$ ) is the result of the large field hysteresis losses. To put our results in perspective, we summarize in Table $\mathrm{I}$ the values of $-\Delta \mathrm{S}_{\mathrm{M}} \max$ and $\mathrm{RC}$ of the present $\mathrm{La}_{0.35} \mathrm{Pr}_{0.275} \mathrm{Ca}_{0.375} \mathrm{MnO}_{3}$ materials and some other known magnetocaloric materials from the literature, within the similar temperature range. Clearly, the $-\Delta \mathrm{S}_{\mathrm{M}}{ }^{\max }$ and $\mathrm{RC}$ values of nanocrystalline $\mathrm{La}_{0.35} \mathrm{Pr}_{0.275} \mathrm{Ca}_{0.375} \mathrm{MnO}_{3}$ are significantly larger than those of the other candidates. The excellent magnetocaloric properties of this sample, in addition to its cost effectiveness, make it an attractive candidate material for use in MEMS and NEMS.

In summary, we have demonstrated the strong enhancement of the magnetocaloric effect and refrigerant capacity and the strong reduction of hysteretic losses in chargeordered $\mathrm{La}_{0.35} \mathrm{Pr}_{0.275} \mathrm{Ca}_{0.375} \mathrm{MnO}_{3}$ at the nanometer scale. The origin of the large magnetocaloric effect is attributed to the collapse of charge ordering and the establishment of ferromagnetic tendency at the surface of the nanoparticles. Our studies open up an opportunity for developing nanoscale magnetic materials for active magnetic refrigerators.

Work at USF was supported by DOE BES Physical Behavior of Materials Program through Grant No. DE-FG0207ER46438. Work at Rutgers was supported by Grant No. DE-FG02-07ER46382. Work at HUT was supported by NAFOSTED through Grant No. 104.02.74.09.

${ }^{1}$ M. H. Phan and S. C. Yu, J. Magn. Magn. Mater. 308, 325 (2007).

${ }^{2}$ T. Samanta, I. Das, and S. Banerjee, Appl. Phys. Lett. 91, 082511 (2007).

${ }^{3}$ S. Gorsse, B. Chevalier, and G. Orveillon, Appl. Phys. Lett. 92, 122501 (2008)

${ }^{4}$ L. Li, K. Nishimura, and H. Yamane, Appl. Phys. Lett. 94, 102509 (2009).

${ }^{5}$ V. Provenzano, A. J. Shapiro, and R. D. Shull, Nature (London) 429, 853 (2004).

${ }^{6}$ N. S. Bingham, M. H. Phan, H. Srikanth, M. A. Torija, and C. Leighton, J. Appl. Phys. 106, 023909 (2009).

${ }^{7}$ L. E. Hueso, P. Sande, D. R. Miguéns, J. Rivas, F. Rivadulla, and M. A. López-Quintela, J. Appl. Phys. 91, 9943 (2002).

${ }^{8}$ P. Poddar, J. Gass, D. J. Rebar, S. Srinath, H. Srikanth, S. A. Morrison, and E. E. Carpenter, J. Magn. Magn. Mater. 307, 227 (2006).

${ }^{9}$ M. H. Phan, M. B. Morales, C. N. Chinnasamy, B. Latha, V. G. Harris, and H. Srikanth, J. Phys. D 42, 115007 (2009).

${ }^{10}$ M. H. Phan, M. B. Morales, N. S. Bingham, H. Srikanth, C. L. Zhang, and S.-W. Cheong, Phys. Rev. B 81, 094413 (2010).

${ }^{11}$ A. L. Lima Sharma, P. A. Sharma, S. K. McCall, S.-B. Kim, and S.-W. Cheong, Appl. Phys. Lett. 95, 092506 (2009).

${ }^{12}$ A. Biswas, T. Samanta, S. Banerjee, and I. Das, Appl. Phys. Lett. 92, 012502 (2008).

${ }^{13}$ A. Biswas, T. Samanta, S. Banerjee, and I. Das, Appl. Phys. Lett. 92, 212502 (2008).

${ }^{14}$ V. S. Kumar and R. Mahendiran, Solid State Commun. 150, 1445 (2010).

${ }^{15}$ S. Karmakar, E. Bose, S. Taran, B. K. Chaudhuri, C. P. Sun, and H. D. Yang, J. Appl. Phys. 103, 023901 (2008). 